Research Article

\title{
Self-Adaptive Particle Swarm Optimization of CT Images for Diagnosis of Severe Traumatic Brain Injury
}

\author{
Zongtao Wu $\mathbb{D},,^{1}$ Jingku Ma $\mathbb{D},{ }^{2}$ Xiaolong Zhou $\mathbb{D},,^{3}$ and Xiumei Ma $\mathbb{D}^{4}$ \\ ${ }^{1}$ Department of Neurosurgery, Ankang Chinese Medicine Hospital, Ankang 725000, Shaanxi, China \\ ${ }^{2}$ Department of Neurosurgery, Shaanxi Provincial Nuclear Industry 215th Hospital, Xianyang 712000, Shaanxi, China \\ ${ }^{3}$ Department of Neurosurgery, Baoji Central Hospital, Baoji 721008, China \\ ${ }^{4}$ Department of Imaging, The First Hospital of Yulin City, Yulin 719000, Shaanxi, China
}

Correspondence should be addressed to Xiumei Ma; 0116007@yzpc.edu.cn

Received 17 May 2021; Revised 20 June 2021; Accepted 3 July 2021; Published 12 July 2021

Academic Editor: Gustavo Ramirez

Copyright ( $\odot 2021$ Zongtao Wu et al. This is an open access article distributed under the Creative Commons Attribution License, which permits unrestricted use, distribution, and reproduction in any medium, provided the original work is properly cited.

\begin{abstract}
This paper aimed to explore the application values of computed tomography (CT) imaging in the treatment of patients with severe traumatic brain injury (STBI), to provide help in the treatment of STBI. In this study, 86 patients with STBI were selected as the research objects and examined by CT based on a self-adaptive particle swarm algorithm (APSO). Besides, patients were treated with hyperbaric oxygen, high-dose hormone shock, and naloxone hydrochloride. The results showed that there was a locally lowdensity brain contusion and laceration injury area, spot-like high-density hemorrhage, and subarachnoid hemorrhage in the images of CT examination. The ventricles of diffuse brain injury were compressed and reduced, and the white matter indicated that the ventricles and cisterns became smaller. Asymmetric hemorrhage and subarachnoid hemorrhage were scattered at the white matter junction. In short, subarachnoid hemorrhage, spot-like high-density hemorrhage at the injury site, and diminished ventricles were typical CT imaging manifestations of STBI. Naloxone hydrochloride method could effectively alleviate the matrix metalloproteinase-9 (MMP-9) $(23.47 \pm 3.45)$ and S100 calcium-binding protein B (S100B) $(0.16 \pm 0.06)$ of patients, which had reliable guiding significance for the later CT examination clinically.
\end{abstract}

\section{Introduction}

Severe traumatic brain injury (STBI) refers to a patient who has suffered severe craniocerebral trauma, immediately showing a deep coma after the injury (often moderate or deep coma), and the coma time is generally greater than 12 hours [1]. Patients will have obvious neurological dysfunction, and their clinical manifestations include paralysis of limbs, loss of sensation, and mental disorders. The patient's vital signs change so that the pulse is weakened, blood pressure is increased, and breathing is slower [2]. However, the literature on STBI is not perfect. Computed tomography (CT) applies the X-rays, $\gamma$-rays, and ultrasound to scan a certain part of the human body together with a highly sensitive detector. It is featured with fast scanning time and clear scanned images [3]. CT examination is the first choice for diagnosing STBI. X-rays are employed to scan the craniocerebral parts of the human body. The detector receives the X-rays at this level and converts them into visible light. Then, the photoelectric signals are converted into electrical signals that are transformed into numbers through the processing of digitizers. The numbers are input into the computer for data processing. The image formation process is equivalent to dividing the selected layer into several rectangular parallelepipeds with the same volume, called voxels [4]. The X-ray absorption coefficient of each voxel is arranged into a digital matrix and stored on the optical disc. Through the digitizer, each number in the digital matrix is converted into black and white different gray squares that are arranged in different matrices to form a CT image [5]. CT can measure the site of STBI in the human body based on different absorption rates and transmittance of X-rays to different tissues, to obtain the data that can be input into the computer to get the cross-sectional image of the brain injury, 
thereby accurately diagnosing the injury location [6]. CT examination has high clinical diagnostic value, can offer accurate diagnosis and treatment images, and provide an effective reference basis for the formulation of treatment plans. When the patient is in a serious condition, an emergency CT examination can quickly scan the patient and accelerate the speed of examination.

At present, brain CT image registration is mainly based on the maximum mutual information measure, combined with an efficient and high-precision optimization search algorithm to achieve. Due to a large amount of maximum mutual information calculation and slow registration speed, the performance of the optimal search algorithm has a great impact on the efficiency and accuracy of registration [7]. Particle swarm optimization (PSO) is an optimal search algorithm proposed in recent years with fast convergence speed and high accuracy. It can improve the registration speed by dynamically controlling the initial population of the particle swarm while maintaining accuracy. Some scholars have summarized the improvement of PSO by domestic and foreign researchers and proposed the evolutionary state estimation (ESE) strategy and the elite learning strategy (ELS). Based on these two strategies, they have proposed a self-adaptive PSO (APSO) [8]. For the registration of brain CT images, APSO selects the appropriate initial population size and problem space range to improve the search accuracy and speed of the algorithm [9]. Some studies have pointed out that when the initial population of APSO is in the range of [2] the optimal solution grows logarithmically; in the range of $(20,+\infty)$, the convergence result has no obvious trend change; in the range of $[2,+\infty)$, with the increase of the initial particle number, the search time increases exponentially. Therefore, while ensuring the search accuracy of the algorithm, it is necessary to control the initial population of the particle swarm to speed up the registration speed. Naloxone hydrochloride is an opioid receptor antagonist, which can competitively antagonize various opioid receptors; clinically, it can improve cerebral circulation and reduce brain injury [10].

In summary, patients with STBI were diagnosed under the APSO-based CT imaging and treated with naloxone hydrochloride and comprehensive treatment measurements. Then, there was an analysis of STBI imaging characteristics during the treatment and the clinical application of CT examination. Therefore, the above would be of great significance for the early diagnosis and improvement of the prognosis of patients with STBI.

\section{Materials and Methods}

2.1. Selection of Research Objects. A total of 86 patients with STBI, who underwent CT examinations in the hospital from January 5, 2019 to June 15, 2020, were selected and divided randomly into a control group and an experimental group. Patients of the control group were $45.8 \pm 8.4$ years old, and the injury factors included external blow damage (6 cases), high-altitude falling (10 cases), traffic accidents (25 cases), and industrial accidents ( 2 cases). Besides, the pupil statuses of patients in the control group included unilateral dilation (31 cases), bilateral dilation ( 6 cases), and sudden increase and decrease (6 cases). There were 43 patients with STBI in the experimental group, with an average age of $44.5 \pm 9.1$ years. The injury factors included external blow damage (5 cases), high-altitude falling (8 cases), traffic accidents (27 cases), and industrial accidents (3 cases). Patients from the experimental group had 3 pupil states, including 29 cases with unilateral pupil dilation, 5 cases with bilateral pupil dilation, and 9 cases with sudden pupil enlargement and reduction. This experiment had been approved by the Ethics Committee of the hospital, and the participating patients or their authorized clients had signed the consent forms for the experimental protocol.

The criteria for inclusion were defined as patients who had the brain CT scanning results meeting the imaging characteristics of STBI, had the points of Glasgow coma score (GCS) that were $\leq 8$ points, had clear experience in violent injury, and had abnormal pupils.

The criteria for exclusion were defined as patients who suffered from other major diseases at the same time, had brain tumors, and had a history of psychiatric diseases.

2.2. Computed Tomography. The patient was examined with a light-speed 16-slice GE CT scanner within the range of 45 minutes to 24 hours of injury. The scanning parameters were as follows. The scanning layer thickness was $10 \mathrm{~mm}$, and the pitch was $0.75-1.5$. The scanning conditions included $512 \times 512$ of scanning matrix, $120 \mathrm{kV}, 190 \mathrm{~mA}, 40$ of the field of view (FOV), and 2 seconds of scanning time. Furthermore, 86 patients were reexamined. After more than two CT examinations, the results could be compared and judged.

2.3. Self-Adaptive Particle Swarm Algorithm. APSO was added with ESE and ELS, so that it had stronger searchability than the previous PSO and could better suppress the local minimum. In the operation of the early algorithm, the particles were scattered in various areas of the space, and the number of population was also scattered. The ESE strategy was proposed based on the best control PSO algorithm. With the continuous progress of the algorithm, the particles began to gradually gather together and converge to the global optimal position. A large number of studies had shown that the particle velocity update weight $W$, the individual particle update coefficient $B_{1}$, and the global optimal position $B_{2}$ all followed different changing laws in the optimization search process, thereby deriving the evolution factor $a$. According to the changing law of the evolution factor $a$, the search process of APSO could be divided into four parts, including exploration, exploitation, aggregation, and jumping out.

The evaluation process of ESE included 3 steps as follows.

Step 1 was to calculate the average distance from a single particle at the current position to all other particles. The distance equation was shown as the following. 


$$
h_{i}=\frac{1}{M-1} \sum_{j=1, j \neq i}^{M} \sqrt{\sum_{k=1}^{F}\left(x_{i}^{k}-x_{j}^{k}\right)^{2}}, \quad i \in[1, N] .
$$

In (1), $h_{i}$ was the average distance from the $i^{\text {th }}$ particle to all other particles, $M$ stood for the population number of particle swarm, and $F$ expressed the dimension of the actual problem.

Step 2 was as follows: $h_{i}$ represented the particle with the best global position, and $h_{g}$ meant the best global position value. $h_{g}$ of all $h_{i}$ was compared to find the maximum $h_{\max }$ and minimum $h_{\min }$ of the average distance from other particles, to calculate the evolution factor $e$, which could be expressed as

$$
a=\frac{h_{g}-h_{\min }}{h_{\max }-h_{\min }} \in[0,1] .
$$

According to the changing law of evolution factor $e$, the search process of APSO could be divided into four stages.

The first one was the exploration stage, as shown in

$$
R_{1}(a) \begin{cases}0, & 0 \leq a \leq 0.4 \\ 5 a-2, & 0.4<a \leq 0.6 \\ 1, & 0.6<a \leq 0.7 \\ -10 a+8, & 0.7<a \leq 0.8, \\ 0, & 0.8<a \leq 1 .\end{cases}
$$

The second one was the exploitation stage, which could be expressed as

$$
R_{2}(a) \begin{cases}0, & 0 \leq a \leq 0.2, \\ 10 a-2, & 0.2<a \leq 0.3 \\ 1, & 0.3<a \leq 0.4 \\ -5 a+3, & 0.4<a \leq 0.6 \\ 0, & 0.6<a \leq 1 .\end{cases}
$$

The third one was the aggregation stage, like

$$
R_{3}(a) \begin{cases}1, & 0 \leq a \leq 0.1, \\ -5 a+1.5, & 0.1<a \leq 0.3, \\ 0, & 0.3<a \leq 1\end{cases}
$$

The fourth one was the jumping-out stage, which was represented in

$$
R_{4}(a) \begin{cases}1, & 0 \leq a \leq 0.7 \\ 5 a-3.5, & 0.7<a \leq 0.9 \\ 1, & 0.9<a \leq 1\end{cases}
$$

Step 3 included the following. The particle update weight $W$, individual update speed coefficient $B_{1}$, and global optimal position update coefficient $B_{2}$ were set based on the evolution state of evolution factor $e$ in step 3. The initial weight $W$ in the algorithm was very small in the exploitation stage and larger in the exploration stage. It did not strictly decrease linearly according to the times of iterations and the time, and its changing law was similar to the law of evolution factor $a$. The law could be expressed as follows:

$$
W(a)=\frac{1}{1+1.5 e^{-2.6 a}} \in[0.4,0.9] \forall a \in[0,1] .
$$

In (7), $W$ and $a$ showed a relationship of S-shaped bending change.

The classic PSO gave the empirical values of the parameters $B_{1}$ and $B_{2}$, namely $B_{1}=B_{2}=2$, and the changes of coefficients $B_{1}$ and $B_{2}$ were controlled by random numbers in the interval $[0,1]$. The solution effect of classical PSO was better for the practical problems such as the small local limit value and search range, but its effect was very poor for practical problems with many local extremes. The equation for the control mode of $B_{1}$ and $B_{2}$ in the APSO was as follows:

$$
\left|b_{i}(g+1)-c_{i}(g)\right| \leq \delta i=1, \quad 2 \delta \in[0.05,0.1] .
$$

In (8), $\delta$ stood for the changing rate of $B_{1}$ and $B_{2}$, which was generally controlled at the interval $[0.05,0.1]$.

$B_{1}$ and $B_{2}$ were for further operation, to obtain $B_{1}+B_{2}=4$, which could be expressed as

$$
b_{i}=\frac{b_{i}}{b_{1}+b_{2}} * 4.0, \quad i=1,2 .
$$

Based on ESE, APSO made the search process of particle swarm optimization to adjust the particle speed update weight $W$, the individual particle update coefficient $B_{1}$, and the global optimal position update coefficient $B_{2}$ according to the above four evolution states. The random search of the classic PSO was improved, and the stability of the algorithm was enhanced.

The evaluation process of ELS included the following.

Assuming that the historical optimal solution vector of the global position was $Q^{d}$, the current worst solution was $Q_{w}^{d}$. Besides, $Q^{d}$ and $Q_{w}^{d}$ were the two attributes of the current solution and historical best solution. The ELS strategy randomly selected a dimension with equal probability to be denoted as $Q^{d}$ that was for mutation by Gaussian distribution, and the equation could be expressed as follows:

$$
Q^{d}=Q^{d}+\left(X_{\max }^{d}-X_{\min }^{d}\right) * \operatorname{Gaussian}\left(\mu, \sigma^{2}\right) .
$$

In (10), $\left[X_{\max }^{d}, X_{\min }^{d}\right]$ stood for the space exploration scope of the actual problem. Gaussian $\left(\mu, \sigma^{2}\right)$ was a Gaussian distribution with mean $\mu=0$ and standard deviation $\sigma$. The Gaussian distribution determined the changing rate of ELS. The equation obtained by $\sigma$ was as follows:

$$
\sigma=\sigma_{\max }-\left(\sigma_{\max }-\sigma_{\min }\right) \frac{g}{G} .
$$

In the ELS process, the historical best solution of $Q^{d}$ was replaced by the result obtained by ELS when the obtained objective function value was better than the historical best solution of $Q^{d}$. If the solution obtained by ELS was between the historical best solutions of $Q^{d}$ and $Q_{w}^{d}$, the individually best solution of $Q_{w}^{d}$ was replaced by 
this value; otherwise, this value replaced the current solution of $Q_{w}^{d}$, and finally, the particle position was updated.

2.4. Treatment Methods and Clinical Effects of Severe Traumatic Brain Injury. After all the research objects were admitted to the hospital, $5 \mathrm{ml}$ of blood was collected from the cubital vein of each patient and stored at room temperature for 1 hour without adding anticoagulants. The specimens were centrifuged at $3,000 \mathrm{r} / \mathrm{min}$ for 5 minutes with the help of corresponding equipment, and the supernatant was aspirated and stored at $-20^{\circ} \mathrm{C}$. An automatic enzyme-linked immunoassay analyzer was applied in this experiment. Besides, the enzyme-linked immunosorbent assay (ELISA) was adopted to detect the protein levels of matrix metalloproteinase-9 (MMP-9) and S100-B in the serum of each patient, and the detection was in strict accordance with the operating procedures of the kit. All patients were given active treatment methods after admission, mainly including treating the injured parts, reducing intracranial pressure, preventing infection, maintaining water and electrolyte balance, and maintaining acid-base balance. Patients of the control group were treated with mild hypothermia, and the patient's body temperature was controlled by a mild hypothermia treatment device and maintained at $32-35^{\circ} \mathrm{C}$. Furthermore, patients of the experimental group were injected with $0.3 \mathrm{mg} \cdot \mathrm{kg}^{-1} \cdot \mathrm{d}^{-1}$ of naloxone hydrochloride with continuous intravenous drip based on the treatment of patients in the control group. The treatment period of patients in the two groups was 7 days.

The clinical efficacy evaluation included 4 types. The first one was healing, namely the disappearance of the patient's clinical symptoms and abnormal vital signs. The second one was the marked effect. It meant that the patient was left with a moderate disability, but could take care of himself or herself. The third one was the effective effect, which indicated the patient was left with a severe disability and was unable to take care of himself or herself. The fourth one was no effect. The patient was in a vegetative state or died. The total effective rate $=$ (the number of patients with healing + the number of patients with marked effect + the number of patients with effective effect)/total number of cases $\times 100 \%$. Finally, there was a comparison of the occurrence of adverse reactions in patients.

2.5. Statistical Methods. In this study, SPSS19.0 statistical analysis software was used for data processing. The calculated data conforming to the normal distribution were expressed by the mean \pm variance $(\bar{x} \pm s)$, and the nonconformity one was represented by the percentage (\%). There were comparisons on basic data of patients, statistics of CT scanning results, the detection of serum MMP-9 and S100-B levels, and the statistical ratio of treatment results. In addition, $P<0.05$ meant that there was a statistically substantial difference.

\section{Results}

3.1. Comparison of Adaptive Particle Swarm Optimization with Basic and Quantum Particle Swarm Optimization Algorithms. According to the simulation experiment in the same environment, the basic PSO (BPSO), quantum PSO (QPSO), and APSO were tested by Sphere, Shaffer, Griewank, and Rastrigin functions. The results of different algorithm test functions to optimize the time showed that the search and positioning time of APSO was faster than that of BPSO and QPSO (Figure 1). Different algorithms were employed to measure the time to search for the optimal solution of the four functions. According to the following image, the optimal solution search and positioning of APSO took less time than that of BPSO and QPSO. Combined with the above results, it indicated that the four test functions were adopted to detect the three algorithms, and APSO had advantages over BPSO and QPSO.

\subsection{Basic Data of Patients with Severe Traumatic Brain Injury.} There were a total of 86 patients with STBI, including 11 cases of external blow damage (12.79\%), 18 cases of highaltitude falling (20.93\%), 52 cases of traffic accidents $(60.46 \%)$, and 5 cases of industrial accidents (5.81\%) (Figure 2).

\subsection{Clinical and CT Imaging Characteristics of Severe Trau-} matic Brain Injury. Craniocerebral injury contained scalp soft tissue injury, skull injury, and intracranial injury. Moreover, the brain tissue injury included brain contusion and laceration, intracerebral hematoma, and diffuse brain injury (Figure 3). Early brain contusion and laceration of brain tissue were displayed as hemorrhage, edema, and necrosis; its clinical manifestations were headache, nausea, vomiting, and disturbance of consciousness; and CT imaging characteristics were locally low-density in the injured area, scattered spot-like high-density hemorrhage, and subarachnoid space hemorrhage, combined with intracerebral hemorrhage, extracerebral hemorrhage, skull fracture, and intracranial gas (Figure 4). Diffuse brain injury included the increased extracellular fluid leading to brain edema, increased intracellular fluid, brain swelling, and brain white matter damage; CT imaging characteristics showed that cerebral edema was compressed or disappeared in the ventricle, and white matter damage was manifested that the ventricles and brain pool became smaller, and asymmetric small focal high-density hemorrhage and subarachnoid hemorrhage were scattered at the white matter junction, and there was no local occupying performance.

\subsection{Comparison of Clinical Efficacy among Patients in the Two} Groups. After patients of the two groups were treated, the total effective rate of patients in the control group was $66.77 \%$, and the rate of patients in the experimental group was $81.39 \%$, indicating that the difference was statistically obvious $(P<0.05)$ (Figure 5). 


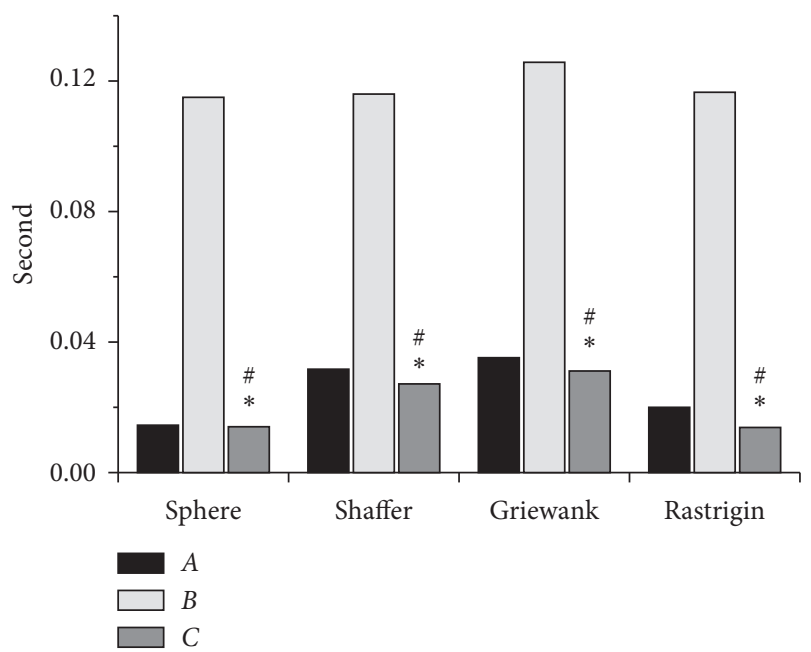

Figure 1: The optimization time of test functions for the three algorithms. Note: $A, B$, and $C$ stand for BPSO, QPSO, and APSO in turn; * denotes that the difference was statistically substantial in contrast to BPSO $(P<0.05)$; and \# indicates that there was a statistically obvious difference in contrast to QPSO $(P<0.05)$.

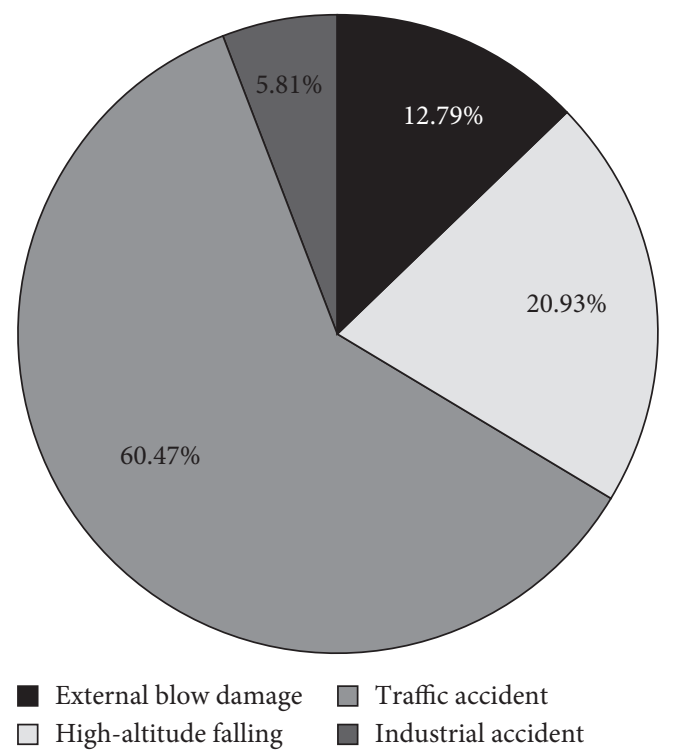

FIgURE 2: Basic data of patients with STBI.

3.5. Diagnostic Results of Computed Tomography Scanning. There were CT diagnosis results of 86 patients with STBI, including 12 cases of scalp soft tissue injury, 9 cases of skull injury, 40 cases of diffuse brain swelling, and 25 cases of brain contusion and laceration (Figure 6). In the images of patients with diffuse brain swelling, the sulci became shallow and the cistern became narrow. Figure 7 shows that the sulcus and cisterns of 13 cases were significantly narrowed, the compression of 17 cases was reduced, and 10 cases suffered from total occlusion (Figure 7). There were 28 cases with unilateral hemisphere swelling, 19 cases with midline structural deviation greater than $6 \mathrm{~mm}$ and less than $10 \mathrm{~mm}$, 9 cases with midline structural deviation greater than $10 \mathrm{~mm}$, and 12 cases with diffuse swelling of the bilateral cerebral hemispheres. In addition, the symmetric

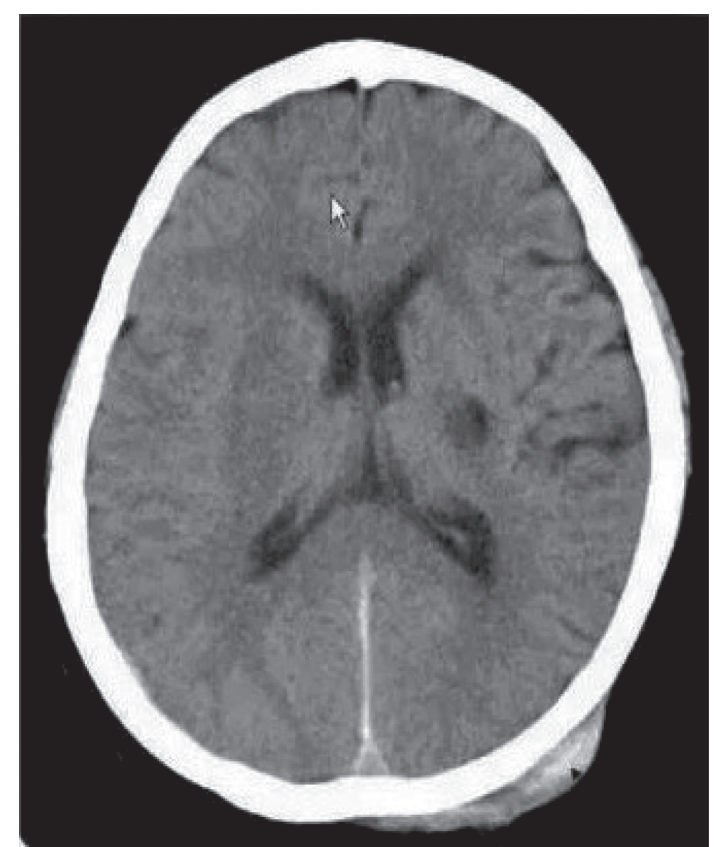

Figure 3: Scalp soft tissue injury.

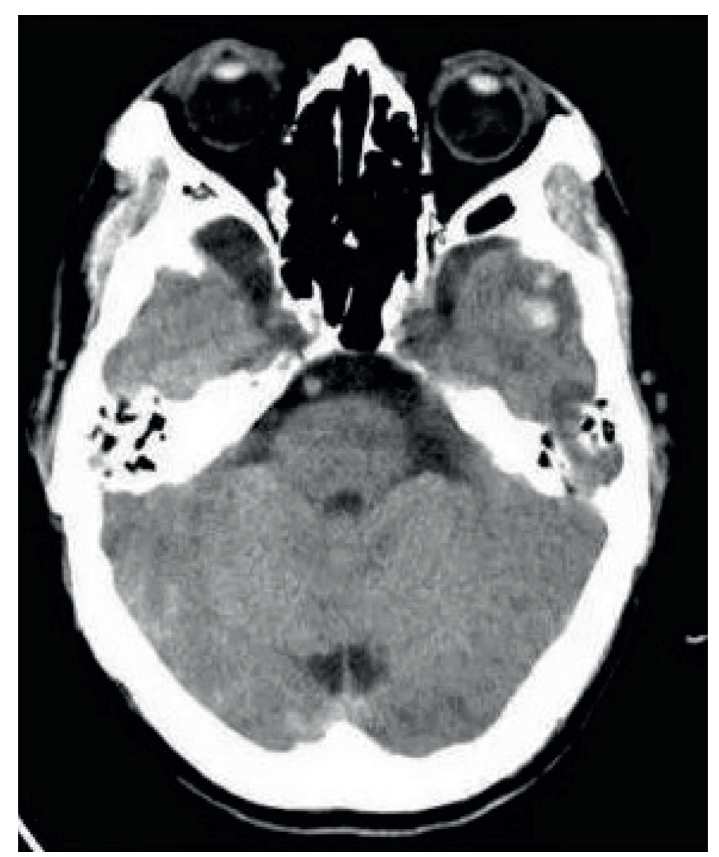

Figure 4: Contusion and laceration of left temporal pole.

compression of the ventricular system was reduced, the sulcus became shallow, the cisterns disappeared, the cortex presented a high-density shadow, and the gyri structure disappeared so that the white matter CT value was abnormal; there was no space-occupying effect in the brain, and the midline structure shift was less than $6 \mathrm{~mm}$ (Figure 8).

3.6. Other Related Computed Tomography Diagnosis Results. In addition to the above diagnosis of patients with STBI, there were 41 cases of subarachnoid rupture, 18 cases of 


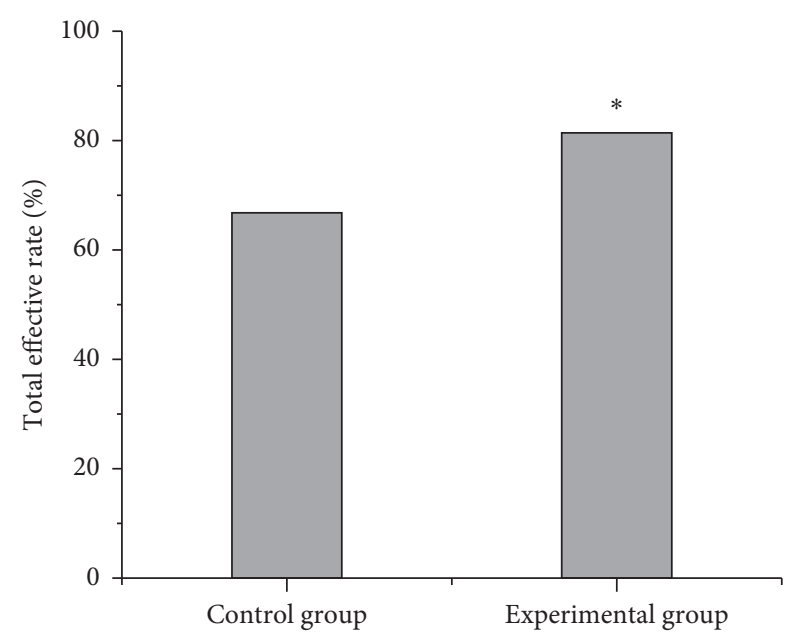

FIgURE 5: Comparison of clinical efficacy among patients in the two groups. Note: ${ }^{*}$ denotes that there was a statistically remarkable difference in contrast to the control group $(P<0.05))$.

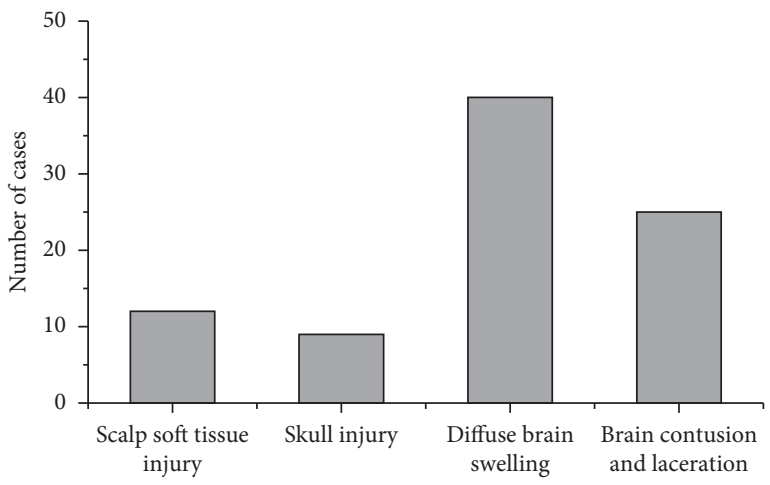

Figure 6: CT examination diagnosis types.

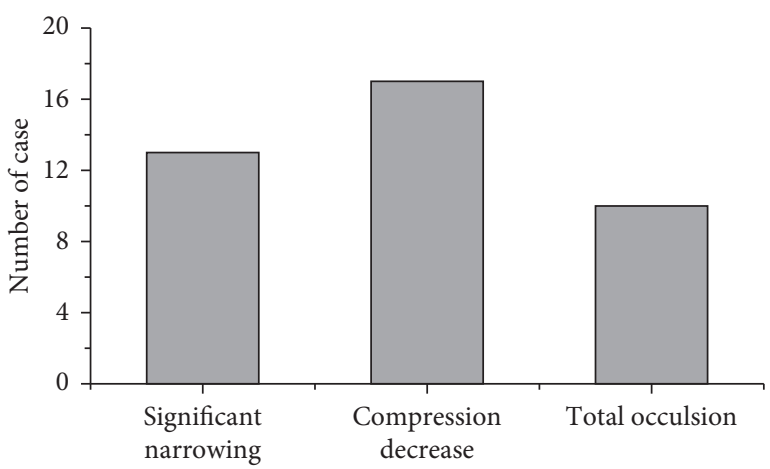

FIGURE 7: CT diagnosis of diffuse brain swelling.

brain herniation, and 43 cases of skull fracture (Figure 9). All patients underwent the surgery. After postoperative reexamination, it was found that the third ventricle and basal cistern of 15 cases gradually changed from small to marked narrowing, and those of 21 cases changed gradually from partial narrowing to complete occlusion. Furthermore, 18

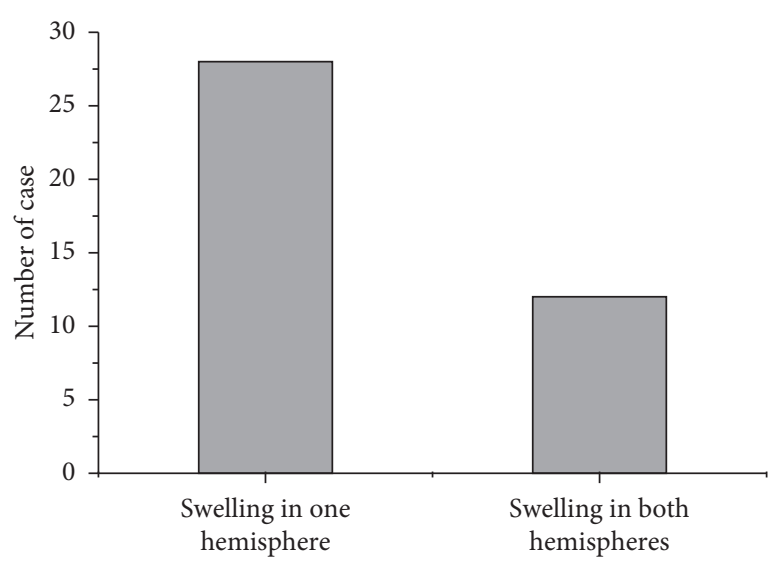

FIgURE 8: CT diagnosis of unilateral and bilateral brain swelling.

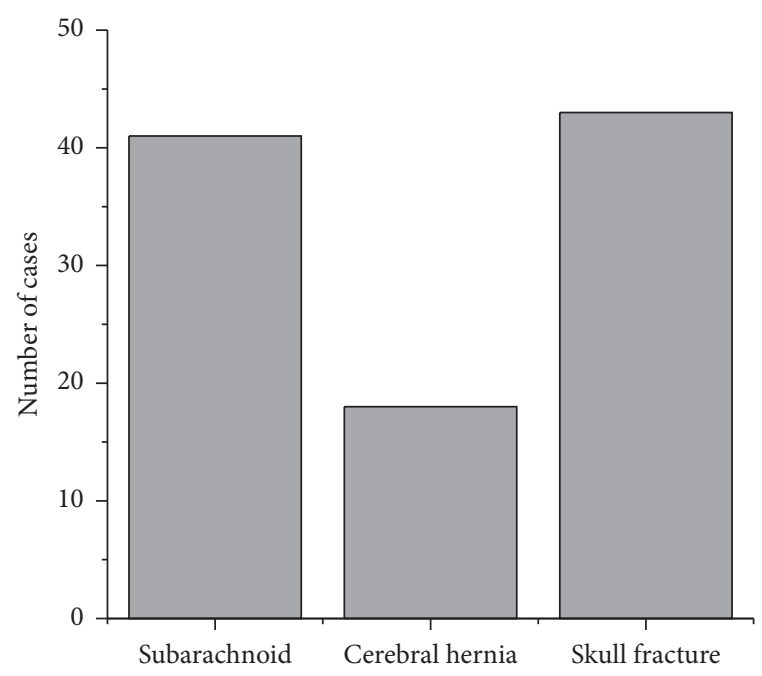

FIgURE 9: Other related CT diagnosis results.

cases were diagnosed for the first time as a brain contusion or intradural hematoma during the reexamination.

\subsection{Comparison of Protein Levels of MMP-9 and S100-B in the} Serum of Patients in the Two Groups. After treatment, the protein levels of MMP-9 and S100-B in the serums of patients from the two groups were sharply lower than those before treatment, and the difference was statistically considerable $(P<0.05)$. By comparing with the control group, the protein levels of MMP-9 $(23.47 \pm 3.45)$ and S100-B $(0.16 \pm 0.06)$ in the serums of patients from the experimental group decreased more obviously after treatment, and there was a statistically great difference $(P<0.05)$ (Figures 10 and 11).

3.8. Treatment Results of All Patients. The total number of patients was 86 , including 75 cases treated with surgery and 11 cases with conservative treatment. Besides, the conditions of 59 patients were improved and the improvement rate was 


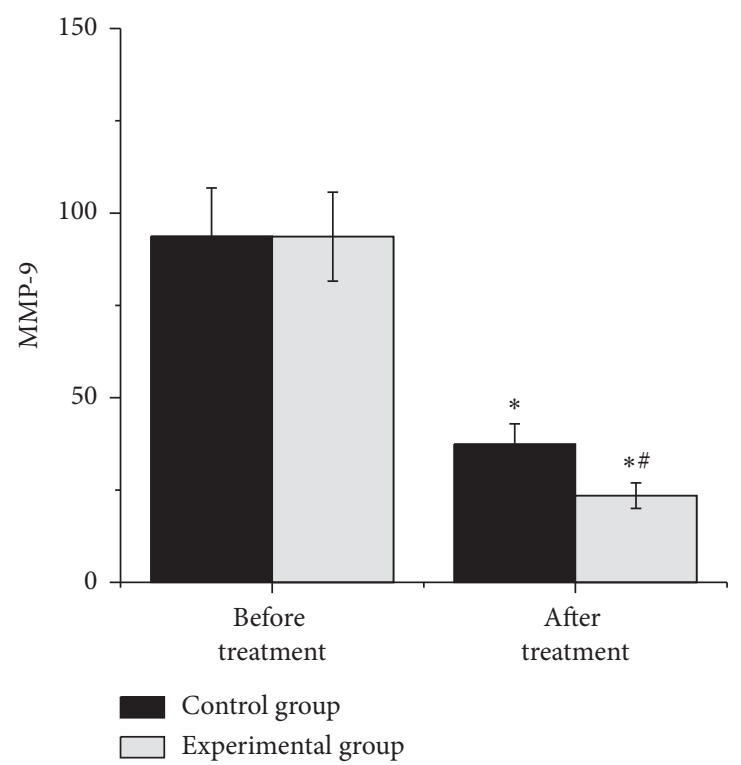

Figure 10: Comparison of MMP-9 levels in patients from the two groups. Note: *indicates there was a statistically huge difference compared with before treatment $(P<0.05)$ and \# reveals that the difference was statistically substantial in contrast to the control group $(P<0.05)$.

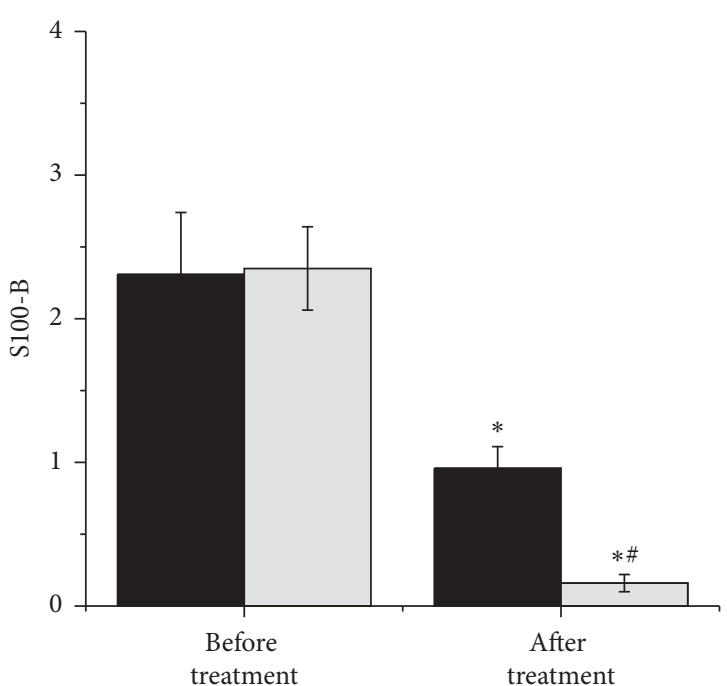

Control group

Experimental group

FIGURE 11: Comparison of the S100-B levels in patients from the two groups. Note: ${ }^{*}$ indicates there was a statistically obvious difference compared with before treatment $(P<0.05)$ and \# reveales that the difference was statistically considerable in contrast to the control group $(P<0.05)$.

$68.6 \%$, and 27 patients died with a mortality rate of $31.39 \%$ (Figure 12).

\section{Discussion}

STBI is a common head injury, and patients will experience loss of consciousness, loss of memory function, and neurological damage [11]. According to the statistics, STBI is one of the critical causes of death of teenagers

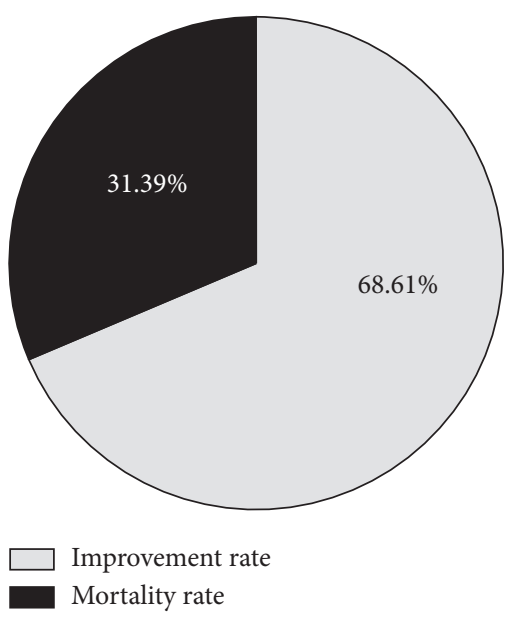

FIGURE 12: The proportion of treatment results of patients with STBI.

from head trauma [12]. CRASH-3 trial collaborators [13] found that the clinical manifestations of STBI included deep coma, abnormal vital signs, and slow breathing immediately after injury, and the recovery cycle of consciousness was long, which was similar to the clinical manifestations of this experiment. The condition of STBI often developed malignantly, and its mortality rate is as high as $80 \%$ clinically. With the continuous progress of China's transportation industry, the incidence of head trauma has a rising trend. In addition, diffuse brain swelling accounts for about $20 \%$ of STBI. Relatively speaking, it is difficult to treat. Falling into a coma for a long time will lead to various complications and increase the postmortality rate [14].

At present, the clinical diagnosis of STBI mainly adopts CT examination. Compared with the image evaluation of 
magnetic resonance imaging (MRI), CT examination takes a short time and can be completed in 5 minutes during emergency examinations, which can accurately determine the degree of craniocerebral injury and save time [15]. CT examination cannot be accurate to the subtentorial lesions of the cerebellum, but it can accurately locate brain hematomas and nonhemorrhagic foci [16]. Besides, it can clearly show skull fractures and accurately diagnose fracture lines and normal cranial lines. It was found in this study that CT images of STBI typically presented the local low-density of brain contusion and laceration, spot-like high-density hemorrhage, and subarachnoid hemorrhage in the injured area. Besides, the ventricle compression of diffuse brain injury decreased or disappeared. Yamada et al. [17] conducted CT impact assessment for patients with STBI, and the manifestations of white matter were smaller ventricle and cerebellum. In this study, the white matter ventricle was smaller, and the junction was scattered with asymmetric hemorrhage and subarachnoid hemorrhage, which were consistent with the above research results. The application of naloxone hydrochloride treatment in a mild hypothermia environment reduced the protein levels of MMP-9 $(23.47 \pm 3.45)$ and S100-B $(0.16 \pm 0.06)$ in the serums of patients with STBI, suggesting that it could greatly improve the conditions of patients with STBI and achieve good clinical effects.

Positive treatment plans should be given to the patients diagnosed with STBI. The main treatment measures included subhyperthermia therapy, hyperbaric oxygen therapy, high-dose hormone shocks, maintaining acid-base balance, maintaining water and electrolyte balance, antiinflammation, and oxygen inhalation. Furthermore, patients with large hematoma and severe cerebral edema in the intracranial cavity should be treated with appropriate surgery.

\section{Conclusion}

CT imaging was constructed based on APSO to diagnose patients with STBI. They were diagnosed and treated comprehensively with naloxone hydrochloride, high-dose hormone shocks, maintaining acid-base balance, water and electrolyte balance, anti-inflammation, and oxygen inhalation. It was found that subarachnoid hemorrhage, spot-like high-density hemorrhage at the injury site, diminished or disappeared ventricle, and the diminished cistern was the typical CT imaging manifestations of patients with STBI. The treatment of naloxone hydrochloride under mild hypothermia could markedly reduce the protein levels of MMP-9 and S100-B in serums of patients, indicating that it can alleviate the condition of patients with STBI. The shortcomings of this experiment were that the number of samples taken is limited and the resulting data might have certain errors. In the future, the sample range should be expanded for further research. To sum up, the CT imaging characteristics were analyzed during the treatment of patients with STBI, which had very crucial clinical value for the early diagnosis of STBI and the improvement of its prognosis.

\section{Data Availability}

No data were used to support this study.

\section{Conflicts of Interest}

The authors declare that they have no conflicts of interest.

\section{References}

[1] R. Firsching, E. Rickels, U. M. Mauer et al., "Guidelines for the treatment of head injury in adults," Journal of Neurological Surgery Part A: Central European Neurosurgery, vol. 78, no. 5, pp. 478-487, 2017.

[2] T. J. Krämer, N. Hack, T. J. Brühl et al., "Depletion of regulatory $\mathrm{T}$ cells increases $\mathrm{T}$ cell brain infiltration, reactive astrogliosis, and interferon- $\gamma$ gene expression in acute experimental traumatic brain injury," Journal of Neuroinflammation, vol. 16, no. 1, p. 163, 2019.

[3] K. Taguchi, "Energy-sensitive photon counting detector-based X-ray computed tomography," Radiol Phys Technol, vol. 10, no. 1, pp. 8-22, 2017.

[4] H. U. M. Shafiq, G. G. Zhang, K. Latifi et al., "Intrinsic dependencies of CT radiomic features on voxel size and number of gray levels," Medical Physics, vol. 44, no. 3, pp. 1050-1062, 2017.

[5] J. Görich and S. Buß, "Computed tomography angiography," Dtsch Arztebl Int, vol. 116, no. 26, p. 460, 2019.

[6] J. N. Useche and S. Bermudez, "Conventional computed tomography and magnetic resonance in brain concussion," Neuroimaging Clinics of North America, vol. 28, no. 1, pp. 15-29, 2018.

[7] Y. Shi, W. K. Wong, J. G. Goldin et al., "Prediction of progression in idiopathic pulmonary fibrosis using CT scans at baseline: a quantum particle swarm optimization-random forest approach," Artificial Intelligence in Medicine, vol. 100, Article ID 101709, 2019.

[8] K. K. Senthil, K. Venkatalakshmi, and K. Karthikeyan, "Lung cancer detection using image segmentation by means of various evolutionary algorithms," Comput Math Methods Med, vol. 2019, Article ID 4909846, 16 pages, 2019.

[9] B. M. Abdel, A. E. Fakhry, H. I. El et al., "Feature and intensity based medical image registration using particle swarm optimization," Journal of Medical Systems, vol. 41, no. 12, 2017.

[10] R. B. Dunne, "Prescribing naloxone for opioid overdose intervention," Pain Management, vol. 8, no. 3, pp. 197-208, 2018.

[11] S. Guo, R. Chen, H. Li et al., "Identify severity bug report with distribution imbalance by CR-SMOTE and ELM," International Journal of Software Engineering and Knowledge Engineering, vol. 29, no. 2, pp. 139-175, 2019.

[12] J. W. Stoneback, A. A. Trizno, and J. C. Albright, "Pediatric and adolescent injury in rodeo," Research in Sports Medicine, vol. 26, no. 1, pp. 114-128, 2018.

[13] Crash-3 trial collaborators, "Effects of tranexamic acid on death, disability, vascular occlusive events and other morbidities in patients with acute traumatic brain injury (CRASH3): a randomised, placebo-controlled trial," Lancet, vol. 394, no. 10210, pp. 1713-1723, 2019.

[14] K. M. Gorse, M. K. Lantzy, E. D. Lee et al., "Transient receptor potential melastatin 4 induces astrocyte swelling but not death after diffuse traumatic brain injury," Journal of Neurotrauma, vol. 35, no. 14, pp. 1694-1704, 2018. 
[15] J. C. Sierink, K. Treskes, M. J. Edwards et al., "REACT-2 study group. Immediate total-body CT scanning versus conventional imaging and selective CT scanning in patients with severe trauma (REACT-2): a randomised controlled trial," Lancet, vol. 388, no. 10045, pp. 673-683, 2016.

[16] S. Majidi, B. Rahim, S. I. Gilani et al., "CT evolution of hematoma and surrounding hypodensity in a cadaveric model of intracerebral hemorrhage," Journal of Neuroimaging, vol. 26, no. 3, pp. 346-350, 2016.

[17] A. Yamada, A. Teramoto, T. Otsuka, K. Kudo, F. Hirishi, and H. Anno, "Preliminary study on the automated skull fracture detection in CT images using black-hat transform," in Proceedings of the 2016 38th Annual International Conference of the IEEE Engineering in Medicine and Biology Society (EMBC), pp. 6437-6440, Orlando, FL, USA, August 2016. 